\title{
Quantum Oscillations in the Surface-Acoustic-Wave Attenuation Caused by a Two-Dimensional Electron System
}

\author{
A. Wixforth and J. P. Kotthaus \\ Institut für Angewandte Physik, 2000 Hamburg 36, Federal Republic of Germany \\ and \\ G. Weimann \\ Forschungsinstitut der Deutschen Bundespost, 6100 Darmstadt, Federal Republic of Germany \\ (Received 25 February 1986)
}

\begin{abstract}
The interaction of surface acoustic waves with a two-dimensional electron system is investigated on $\mathrm{GaAs} / \mathrm{Ga}_{1-x} \mathrm{Al}_{x} \mathrm{As}$ heterojunctions at low temperatures $(T \leqslant 4.2 \mathrm{~K})$ and high magnetic fields $(\leqslant 10 \mathrm{~T})$. Surface acoustic waves are propagated through the heterojunction containing a highmobility two-dimensional electron gas and situated between the emitting and receiving transducers. The interaction between the two-dimensional electron system and the surface acoustic wave produces strong quantum oscillations in the sound attenuation and the sound velocity which can be quantitatively explained.
\end{abstract}

PACS numbers: $73.40 . \mathrm{Lq}, 72.50 .+\mathrm{b}, 73.90 .+\mathrm{f}$

The electron-phonon interaction in quasi twodimensional electron systems (2DES) in metal-oxidesemiconductor or semiconductor heterostructures has attracted much attention in recent years. The interaction between optical phonons and a 2DES has been studied via magnetophonon resonances ${ }^{1}$ and the resonant magnetopolaron observed in cyclotron resonance experiments. ${ }^{2,3}$ The theoretical description of this interaction is rather advanced. ${ }^{4}$ Similarly successful has been the investigation of the interaction between ballistic acoustic phonons and a 2DES via the deformation potential. ${ }^{5}$ Here we report a first experimental study of the influence of a 2DES in a piezoelectric semiconductor structure on the propagation of coherent surface acoustic waves (SAW). The experiments show that the complete quantization of a 2DES in a strong magnetic field causes giant quantum oscillations in the SAW attenuation reflecting the characteristic Shubnikov-de Haas oscillations of the conductivity in a $2 \mathrm{DES}^{6}{ }^{6}$ We believe that our experiments not only are of fundamental interest but also may provide the basis for novel devices using acoustoelectric phenomena in a $2 \mathrm{DES}$.

A sketch of the sample and the experimental configuration is given in Fig. 1. The sample is a GaAs/ $\mathrm{Ga}_{1-x} \mathrm{Al}_{x} \mathrm{As} \quad(x=0.37)$ heterojunction grown by molecular beam epitaxy on a $\mathrm{Cr}$-doped semi-insulating GaAs $(100)$ substrate. The single-interface heterojunction consists of a $1-\mu \mathrm{m}$-thick, unintentionally doped, $p$-type GaAs layer $\left(N_{A}=2 \times 10^{14} \mathrm{~cm}^{-3}\right)$, a 20 -nmthick undoped $\mathrm{Ga}_{1-x} \mathrm{Al}_{x}$ As spacer, a 35-nm-thick $n$ type $\mathrm{Ga}_{1-x} \mathrm{Al}_{x}$ As layer doped with $\mathrm{Si}$ to a free-carrier concentration of $1.2 \times 10^{18} \mathrm{~cm}^{-3}$, and a 20 -nm-thick GaAs cover layer. At $4.2 \mathrm{~K}$ the heterojunction contains electrons at a concentration of $3.2 \times 10^{11} \mathrm{~cm}^{-2}$ with a zero-field mobility of $\mu=517000 \mathrm{~cm}^{2} / \mathrm{V} \mathrm{sec}$.
Except for an area of $2 \times 4.5 \mathrm{~mm}^{2}$ in the center of the sample, the $\mathrm{Ga}_{1-x} \mathrm{Al}_{x}$ As is chemically removed. Surface acoustic waves are generated and detected at a fundamental frequency of about $70 \mathrm{MHz}$ with standard interdigital transducers which are prepared directly onto the GaAs substrate on both sides of the central area carrying the 2DES. SAW pulses of typically 1$\mu \mathrm{sec}$ duration are propagated along the [011] direction and analyzed in amplitude and phase by use of homodyne detection and standard boxcar integration techniques. $^{7}$ The sample is located at the center of a superconducting solenoid providing fields up to $12 \mathrm{~T}$ perpendicular to the sample surface.

The magnetic field dependences of the detected SAW intensity and phase, measured at sufficiently low SAW amplitudes to avoid nonlinear effects, ${ }^{8}$ are shown in Figs. 2(a) and 2(b), respectively. For comparison Fig. 2(c) displays the magnetic field dependence of the conductivity $\sigma_{x x}(B)$ in the 2DES as extracted from measurements of $\rho_{x x}(B)$ and $\rho_{x y}(B)$,

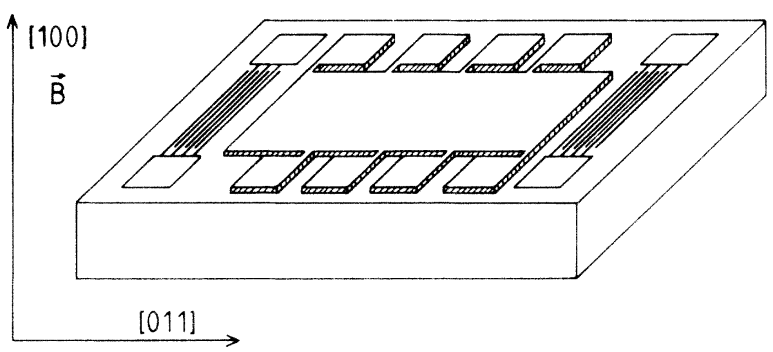

FIG. 1. Sketch of the GaAs/ $\mathrm{Ga}_{1-x} \mathrm{Al}_{x}$ As sample with interdigital transducers $(\lambda \simeq 39 \mu \mathrm{m}, 10$ finger pairs $)$ and $\mathrm{dc}$ contact pads. The heterojunction layer is chemically removed except for the area indicated between the transducers. 


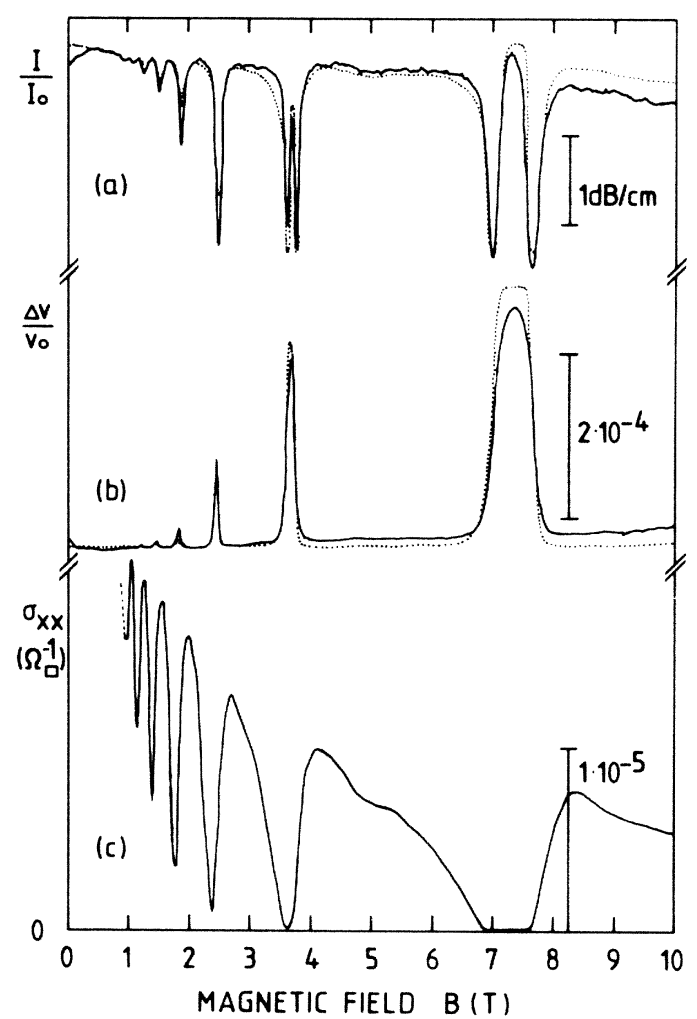

FIG. 2. Magnetic field dependence of (a) the SAW intensity $I / I_{0}$ and (b) phase after transmission through the single-layer heterojunction at $T=4.2 \mathrm{~K}$. The phase signal is calibrated in units of velocity change $\Delta v / v_{0}$. (c) The conductivity $\sigma_{x x}$ as extracted from measurements on a reference Hall bar fabricated on the same wafer. Since $\sigma_{x x}$ increases dramatically with decreasing $B$ below $1 \mathrm{~T}$ and is $\sigma(B=0)=2.6 \times 10^{-2}(\Omega / \square)^{-1}$, this region has been omitted for clarity. The dotted lines in (a) and (b) indicate a fit according to Eqs. (1) and (2) using $\sigma_{x x}$ from (c), $\sigma_{M}=4 \times 10^{-7}(\Omega / \square)^{-1}$, and $\alpha=3.2 \times 10^{-4}$.

measured on a Hall-bar sample fabricated on the same wafer. At low magnetic fields $(B \leq 1 \mathrm{~T})$ the SAW intensity and phase are nearly constant, whereas $\sigma_{x x}(B)$ drops dramatically as expected for a high-mobility heterojunction. For $B>1 \mathrm{~T}$ both the SAW intensity, or equivalently the attenuation, and phase exhibit characteristic structures which coincide in position with the Shubnikov-de Haas oscillations of $\sigma_{x x}(B)$. One remarkable feature of the quantum oscillations in the SAW attenuation is that they appear to split at higher magnetic fields whereas no such splitting is observed in either the phase signal or the conductivity $\sigma_{x x}(B)$ of the 2DES. As it turns out, the occurrence of this splitting yields a sensitive test for a theoretical description of the magnetic field dependence of the SAW attenuation as does the fact that the magneticfield-induced SAW attenuation appears not to exceed a value of $\Gamma=2.2 \mathrm{~dB} / \mathrm{cm}$.

The interaction of the SAW with the 2DES can be described as a relaxation-type interaction of the polari- zation field, produced by the SAW and propagating with phase velocity $v$, with the conductivity $\sigma_{x x}$ of the two-dimensional sheet of charge situated at the heterojunction interface. ${ }^{8-10}$ Since here the acoustic wavelength $\lambda=2 \pi / k$ is large compared with the thickness of the heterojunction as well as the Fermi wavelength $\lambda_{\mathrm{F}}$ and the mean free path $l=v_{\mathrm{F}} \tau$ of the carriers in the 2DES, one can neglect the acoustic loading by the $\mathrm{Ga}_{1-x} \mathrm{Al}_{x}$ As layer as well as nonlocal effects. In the weak-coupling limit and with neglect of diffusion effects, one obtains for the attenuation coefficient $\Gamma$ and the change in SAW velocity normalized to the velocity $v_{0}=v(\sigma \rightarrow \infty)$, respectively, ${ }^{8-10}$

$$
\Gamma=\frac{\alpha k\left(\sigma_{x x} / \sigma_{m}\right)}{1+\left(\sigma_{x x} / \sigma_{m}\right)^{2}}, \quad \sigma_{m}=v\left(\epsilon_{0}+\epsilon_{s}\right)
$$

and

$$
\frac{\Delta v}{v_{0}}=\frac{v\left(\sigma_{x x}\right)-v_{0}}{v_{0}}=\frac{\alpha}{1+\left(\sigma_{x x} / \sigma_{m}\right)^{2}} .
$$

Here $\alpha$ is an effective piezoelectric coupling coefficient and $\epsilon_{0}$ and $\epsilon_{s}$ are the dielectric constants of the vacuum and the semiconductor. Using recent literature values for the [011] direction of a (100) GaAs surface $^{11}$ one expects $\alpha=3.6 \times 10^{-4}$ and $\sigma_{m}=3.3 \times 10^{-7}$ $(\Omega / \square)^{-1}$. Equation (1) predicts that with decreasing conductivity $\sigma_{x x}$ the SAW attenuation increases until a maximum is reached when $\sigma_{x x}=\sigma_{m}$. For even lower values of $\sigma_{x x} \Gamma$ decreases again. This is in good agreement with the experimental observation. For a given quantum oscillation in $\sigma_{x x}(B)$, where $\sigma_{x x}(B)$ drops below $\sigma_{m}$, e.g., the one around $B \simeq 7 \mathrm{~T}$, the sound attenuation reaches two maxima with increasing $B$ as predicted by Eq. (1) if one uses $\sigma_{x x}=\sigma_{x x}(B)$. The magnetic field dependence of the observed intensity $I / I_{0}$ and velocity change $\Delta v / v_{0}$ are well described by Eqs. (1) and (2), respectively, if we use the experimental $\sigma_{x x}(B)$ [Fig. 2(c)] and $\alpha$ and $\sigma_{m}$ as fit parameters. The results are entered as the dotted line in Figs. 2 (a) and 2(b), respectively. This fit to the experimental data yields $\sigma_{m}=4 \times 10^{-7}(\Omega / \square)^{-1}$ and $\alpha$ $=3.2 \times 10^{-4}$. Both values agree reasonably well with the ones predicted above with use of data from a pure $\mathrm{GaAs}$ surface. However, there are minor differences between the experimental observation and the simple theory that are consistently observed. For example, at low magnetic field we observe a change in $I / I_{0}$ and $\Delta v / v_{0}$ that is not predicted by the above theory. For a given quantum oscillation that exhibits splitting, the low-field minimum in $I / I_{0}$ is always smaller than the high-field minimum. The maximum attenuation also seems to increase with magnetic field. To describe these observations a more refined theoretical model is needed. If, at a given $B$, we vary $\sigma_{x x}(B)$ either by changing the sample temperature or by changing the carrier density $n_{s}$ via illumation with band-gap radia- 
tion, Eqs. (1) and (2) qualitatively correctly predict the observed changes of the quantum oscillations in the SAW attenuation and phase.

At higher SAW intensities, as in Fig. 2, we observe changes in the SAW attenuation that are consistent with carrier heating in the 2DES by the SAW. Figure 3 illustrates these power-dependent effects for the quantum oscillation in the intensity occurring at around $3.7 \mathrm{~T}$. With increasing SAW power the maximum in $I / I_{0}$ occurring when $\sigma_{x x}(B)$ is very low $\left[\sigma_{x x}(B)<<\sigma_{m}\right]$ is decreasing whereas the double minima in $I / I_{0}$ decrease their separation on the $B$ scale until they merge at high SAW intensities $I_{0}$. To relate these changes in $\Gamma$ with changes in $\sigma_{x x}(B)$ we have measured the change of $\sigma_{x x}(B)$ at the highest power level in Fig. 3 in a time-resolved pulsed experiment, using the contact pads on the sample. We use short SAW pulses $(\sim 1 \mu \mathrm{sec})$ and a low repetition rate $(-100 \mathrm{~Hz})$ to avoid thermal heating of the whole sample. The change that is observed in $\sigma_{x x}(B)$ by application of the high-power rf pulse is $\Delta \sigma_{x x}(B)$ $=2 \times 10^{-7}(\Omega / \square)^{-1}$ and consistent with the change measured in $\Gamma(B)$. Within the time resolution of the experiment $(\sim 1 \mu \mathrm{sec})$ the response in $\sigma_{x x}(B)$ to the SAW pulse is instantaneous. We thus conclude that at high power levels the SAW pulse causes carrier heating in the 2 DES which decreases the mobility ${ }^{12}$ and thus the minima in $\sigma_{x x}(B)$. This "phonoconductive" response in turn decreases the minima in the SAW at-

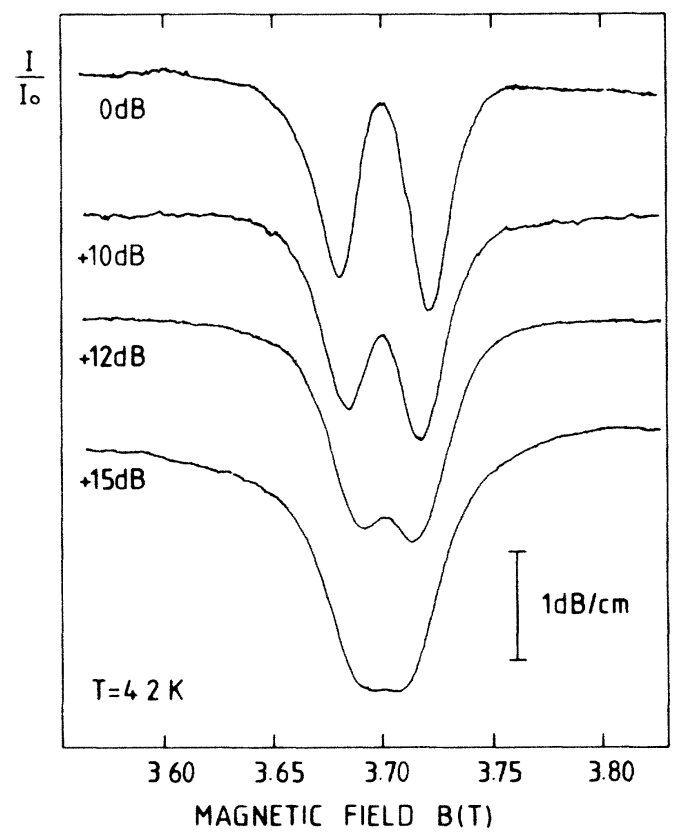

FIG. 3. Power dependence of the SAW intensity for the quantum oscillation at $B=3.7 \mathrm{~T}$. At the lowest level the electrical power coupled into the transducer is about 340 $\mathrm{mW} / \mathrm{cm}^{2}$. With increasing power the splitting of the structure vanishes, as the two minima merge. tenuation. At these high-power levels we also detect a magnetic-field-dependent voltage between two contact pads situated along the long side of the sample, when source and drain contacts are shorted. This indicates the occurrence of an acoustoelectric current in the $2 \mathrm{DES}^{8}$ and will be discussed in detail elsewhere.

In summary, we have observed quantum oscillation in the SAW attenuation and phase which are caused by the interaction of the SAW with a 2DES. The interaction is well described by a simple theory in the smallsignal limit. At large SAW intensities we observe SAW-induced changes in the conductivity as well as saturation effects in the SAW attenuation, both of which can be explained by hot-electron effects. Our experiments demonstrate that SAW measurements are a sensitive probe of changes in the magnetoconductivity of a 2DES at magnetic fields, where $\sigma_{x x}(B)$ becomes very small. Since the occurrence of $\sigma_{x x}(B) \approx 0$ correlates with the occurrence of plateaus in $\sigma_{x y}(B)$, i.e., the quantum Hall effect, ${ }^{13} \mathrm{SAW}$ studies may prove valuable for a better understanding of the quantum Hall effect. One can also expect novel phenomena in the propagation of SAW through a $2 \mathrm{DES}$, when the electron drift velocity $v_{D}$ exceeds the SAW velocity or when the electron mean free path $l$ exceeds the SAW wavelength $\lambda$. Thus far the SAW experiments reported here mark only the beginning of a novel area of experiments on semiconductor structures with a 2DES and may eventually result in new acoustoelectric devices employing a 2 DES.

We wish to thank A. V. Chaplik and B. Lüthi for stimulating discussions. The financial support of the Deutsche Forschungsgemeinschaft is gratefully acknowledged.

${ }^{1}$ D. C. Tsui, Th. Englert, A. Y. Cho, and A. C. Gossard, Phys. Rev. Lett. 44, 341 (1980).

${ }^{2}$ M. Horst, U. Merkt, and J. P. Kotthaus, Phys. Rev. Lett. 50, 754 (1983).

${ }^{3}$ R. J. Nicholas, C. C. Brunel, S. Huant, K. Karrai, J. C. Portal, M. A. Brummell, M. Razeghi, K. Y. Cheng, and A. Y. Cho, Phys. Rev. Lett. 55, 883 (1985).

${ }^{4}$ See. e.g., S. Das Sarma and B. A. Mason, Ann. Phys. (N.Y.) 163, 78 (1985).

5 J. C. Hensel, B. I. Halperin, and R. C. Dynes, Phys. Rev. Lett. 51, 2302 (1983).

${ }^{6}$ A. B. Fowler, F. F. Fang, W. E. Howard, and P. J. Stiles, Phys. Rev. Lett. 16, 901 (1966).

${ }^{7}$ See, e.g., J. Heil, I. Kouroudis, B. Lüthi, and P. Thalmeier, J. Phys. C 17, 2433 (1984).

${ }^{8}$ K. A. Ingebrigtsen, J. Appl. Phys. 41, 454 (1970).

${ }^{9}$ P. Bierbaum, Appl. Phys. Lett. 21, 595 (1972).

${ }^{10}$ A. V. Chaplik, Sov. Tech. Phys. Lett. 10, 584 (1984).

${ }^{11}$ T. W. Grudkowski and M. Gilden, Appl. Phys. Lett. 38, $412(1980)$.

${ }^{12}$ W. Walukiewicz, H. E. Ruda, J. Lagowski, and H. C. Gatos, Phys. Rev. B 30, 4571 (1984).

${ }^{13} \mathrm{~K}$. von Klitzing, G. Dorda, and M. Pepper, Phys. Rev. Lett. 45, 494 (1980). 